\title{
Delay-Dependent Stability Criteria for Continuous Systems with Two Additive Interval Time-Varying Delays and Nonlinear Perturbations
}

\author{
Jianmin Jiao and Xiaojun Sun \\ Institute of Mathematics and Information Science, Baoji University of \\ Arts and Sciences, Baoji, 721013, China \\ jmjiao@126.com
}

\begin{abstract}
In this paper, the problem of stability analysis for continuous systems with two additive interval time-varying delays and nonlinear perturbations is investigated. By constructing an appropriate Lyapunov functional and using reciprocally convex technique, a delayrange-dependent stability criterion is obtained. Based on this criterion, some less conservative and simplified stability criteria for linear systems with two additive timevarying delays are proposed. All the obtained criteria are expressed as a set of linear matrix inequalities, which can be readily tested by using standard numerical software. Finally, two numerical examples are employed to illustrate the effectiveness of the obtained theoretical results.
\end{abstract}

Keywords: Delay-dependent stability, Additive interval time-varying delays, Nonlinear perturbations, Linear matrix inequality (LMI)

\section{Introduction}

Time delay is a common phenomenon which exists in many practical systems, such as biological systems, chemical systems, electronic systems, network control systems, and it is regarded as a major source of instability and poor performance [1]. Since systems stability is an essential requirement in many applications, much effort has been made to investigate stability problem for various time-delay systems over the past years. For recent progress, refer to [2-10] and the references therein. The most commonly and frequently used state-space model of time-delay systems contain one single delay in the state. Recently, a new model for continuous systems with two additive time-varying delay components was proposed in [11]. The new model has a strong application background in networked control systems. Some delay-dependent stability results for linear systems with two additive time-varying delays have been reported. In [11], Lam et.al. have initially investigated the stability for this kind of systems, and a stability criterion was obtained. In [12-13], by constructing new Lyapunov functional, some improved delay-dependent stability criteria were derived. In [14], by taking more information of the time-varying delays in Lyapunov functional into account and by utilizing free matrix variables in approximating certain integral terms, a robust stability criterion for uncertain linear systems with two additive time-varying delays was given. In [15], by defining a more general Lyapunov functional and employing convex polyhedron method to estimate the upper bound on the time derivative of the Lyapunov functional, an improved delaydependent stability criterion is established. In [16], by using a simple type of Lyapunov functional, some delay-dependent stability criteria for singular system with two additive time-varying delays were established with fewer matrix variables. Very recently, by taking advantage of integral inequalities and new Lyapunov functional, some new less conservative delay-dependent stability criteria are presented in [17-19]. Yet it is worth 
pointing out that, though the works above were elegant, there still exist some points waiting for the improvement. Firstly, the systems considered in [11-19] are all linear systems and ranges of time-varying delays are from zero to an upper bound, while little literature research the stability problem for systems with additive interval time-varying delays and nonlinear perturbations. Secondly, many matrix variables are introduced in the existing research result, which brings a large computational burden. Thirdly, the constructions of Lyapunov functional and analyzed techniques employed above still need to be improved, and there is room for further investigation.

Motivated by the preceding discussions, this paper deals with delay-dependent stability problem for continuous systems with two additive interval time-varying delays and nonlinear perturbations. By introducing a new Lyapunov functional and using reciprocally convex technique, a delay-range-dependent stability criteria is derived. Furthermore, some less conservative and simplified stability criteria for linear systems with two additive time-varying delays are proposed. Finally, numerical examples are given to illustrate the effectiveness of the obtained criteria.

Notations: Throughout this paper, $\mathbf{R}^{n}$ denotes the $n$-dimensional Euclidean space, while $\mathbf{R}^{m \times n}$ refers to the set of all real matrices with $m$ rows and $n$ columns. $A^{\mathrm{T}}$ represents the transpose of the matrix $A$. For real symmetric matrices $X$ and $Y$, the notation $X \geq Y$ (respectively, $X>Y$ ) means matrix $X-Y$ is positive-semidefinite

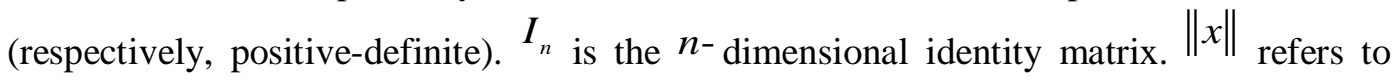
the Euclidean norm of the vector $x$, that is $\|x\|=\sqrt{x^{\mathrm{T}} x}$.

\section{Problem Description and Preliminaries}

Consider the following continuous system with two additive interval time-varying delays and nonlinear perturbations

$$
\left\{\begin{array}{l}
\dot{x}=A x(t)+B x\left(t-d_{1}(t)-d_{2}(t)\right)+f(t, x(t))+g\left(t, x\left(t-d_{1}(t)-d_{2}(t)\right)\right), \\
x(t)=\varphi(t), t \in\left[-\left(d_{12}+d_{22}\right), 0\right],
\end{array}\right.
$$

where $x(t) \in \mathbf{R}^{n}$ is the state vector, $\varphi(t) \in \mathbf{R}^{n}$ is a continuous vector valued initial function. $A, B \in \mathbf{R}^{n \times n}$ are constant matrices. $d_{1}(t)$ and $d_{2}(t)$ represent the two delay components in the state and satisfy following conditions

$$
d_{i 1} \leq d_{i}(t) \leq d_{i 2}, \dot{d}(t) \leq \mu_{i},(i=1,2)
$$

where $0 \leq d_{i 1}<d_{i 2}, 0 \leq \mu_{i}<1(i=1,2)$ are known constants. $f(t, x(t))$ and $g\left(t, x\left(t-d_{1}(t)-d_{2}(t)\right)\right)$ are the nonlinear perturbations with respect to current state and additive delayed state, they satisfy $f(t, 0)=g(t, 0)=0$ and following bounding conditions

$$
\begin{gathered}
f^{\mathrm{T}}(t, x(t)) f(t, x(t)) \leq \alpha^{2} x^{\mathrm{T}}(t) F^{\mathrm{T}} F x(t), \\
g^{\mathrm{T}}\left(t, x\left(t-d_{1}(t)-d_{2}(t)\right)\right) g\left(t, x\left(t-d_{1}(t)-d_{2}(t)\right)\right) \\
\leq \beta^{2} x^{\mathrm{T}}\left(t-d_{1}(t)-d_{2}(t)\right) G^{\mathrm{T}} G x\left(t-d_{1}(t)-d_{2}(t)\right),
\end{gathered}
$$

where $F$ and $G$ are known constant matrices with appropriate dimensions, $\alpha \geq 0$ and $\beta \geq 0$ are known scalars.

The Jensen inequality and reciprocally convex lemma will be useful later, so they are listed as the following lemmas. 
Lemma 1 (Jensen inequality [1]) For any matrix $R>0$, scalars $\gamma_{1}$ and $\gamma_{2}$ satisfying $\gamma_{2}>\gamma_{1}$, a vector function $\omega:\left[\gamma_{1}, \gamma_{2}\right] \rightarrow \mathbf{R}^{n}$ such that the integrations concerned are well defined, then the following inequality holds

$$
-\left(\gamma_{2}-\gamma_{1}\right) \int_{\gamma_{1}}^{\gamma_{2}} \omega^{\mathrm{T}}(s) R \omega(s) \mathrm{d} s \leq-\int_{\gamma_{1}}^{\gamma_{2}} \omega^{\mathrm{T}}(s) \mathrm{d} s R \int_{\gamma_{1}}^{\gamma_{2}} \omega(s) \mathrm{d} s .
$$

Lemma 2 (Reciprocally convex lemma [5]) For any real numbers $\gamma_{1}>0, \gamma_{2}>0$ with $\gamma_{1}+\gamma_{2}=1$, vectors $\zeta_{1}, \zeta_{1}$, and matrices $R>0, M$ satisfying

then the following inequality holds

$$
\left[\begin{array}{cc}
R & M \\
M^{\mathrm{T}} & R
\end{array}\right] \geq 0
$$

$$
-\frac{1}{\gamma_{1}} \zeta_{1}^{\mathrm{T}} R \zeta_{1}-\frac{1}{\gamma_{2}} \zeta_{2}^{\mathrm{T}} R \zeta_{2} \leq-\left[\begin{array}{c}
\zeta_{1} \\
\zeta_{2}
\end{array}\right]^{\mathrm{T}}\left[\begin{array}{cc}
R & M \\
M^{\mathrm{T}} & R
\end{array}\right]\left[\begin{array}{l}
\zeta_{1} \\
\zeta_{2}
\end{array}\right]
$$

\section{Main results}

Before introducing the main results, following notations are defined for simplicity.

$$
\begin{gathered}
f=f(t, x(t)) \\
g=g\left(t, x\left(t-d_{1}(t)-d_{2}(t)\right)\right) \\
\xi(t)=\left[\begin{array}{cccc}
x^{\mathrm{T}}(t) & x^{\mathrm{T}}\left(t-d_{11}-d_{21}\right) & x^{\mathrm{T}}\left(t-d_{1}(t)-d_{21}\right) & x^{\mathrm{T}}\left(t-d_{1}(t)-d_{2}(t)\right) \\
x^{\mathrm{T}}\left(t-d_{1}(t)-d_{22}\right) & x^{\mathrm{T}}\left(t-d_{12}-d_{22}\right) & f^{\mathrm{T}} & g^{\mathrm{T}}
\end{array}\right]^{\mathrm{T}} .
\end{gathered}
$$

Now, we provide a delay-range-dependent stability criterion for system (1) with interval time-varying delays $d_{1}(t)$ and $d_{2}(t)$ satisfying (2) and nonlinear perturbations $f$ and $g$ satisfying (3) and (4).

Theorem 1 For given scalars $0 \leq d_{i 1}<d_{i 2}, 0 \leq \mu_{i}<1(i=1,2), \alpha \geq 0$ and $\beta \geq 0$, the system (1) with two additive interval time-varying delays $d_{1}(t)$ and $d_{2}(t)$ satisfying (2) and nonlinear perturbations $f$ and ${ }^{g}$ satisfying (3) and (4) is stable, if there exist matrices $P>0, Q_{i}>0(i=1,2, \cdots, 5), R_{j}>0, M_{j}(i=1,2)$, and scalars $\varepsilon_{1} \geq 0$, $\varepsilon_{2} \geq 0$ such that the following LMIs hold

$$
\begin{gathered}
\Xi<0, \\
{\left[\begin{array}{cc}
R_{2} & M_{i} \\
M_{i}^{\mathrm{T}} & R_{2}
\end{array}\right] \geq 0(i=1,2),}
\end{gathered}
$$

where 


$$
\begin{aligned}
\Xi & =e_{0}{ }^{\mathrm{T}} \tilde{R} e_{0}+e_{0}{ }^{\mathrm{T}} P e_{1}+e_{1}^{\mathrm{T}} P e_{0}+e_{1}^{\mathrm{T}}\left(Q_{1}+Q_{2}+Q_{3}+Q_{4}+Q_{5}\right) e_{1}+e_{1}^{\mathrm{T}}\left(\varepsilon_{1} \alpha^{2} F^{\mathrm{T}} F\right) e_{1} \\
& -\left(e_{1}-e_{2}\right)^{\mathrm{T}} R_{1}\left(e_{1}-e_{2}\right)-e_{2}{ }^{\mathrm{T}} Q_{1} e_{2}-\left(1-\mu_{1}\right) e_{3}{ }^{\mathrm{T}} Q_{2} e_{3}-\left(1-\mu_{1}-\mu_{2}\right) e_{4}{ }^{\mathrm{T}} Q_{3} e_{4} \\
& +e_{4}{ }^{\mathrm{T}}\left(\varepsilon_{2} \beta^{2} G^{\mathrm{T}} G\right) e_{4}-\left(1-\mu_{1}\right) e_{5}{ }^{\mathrm{T}} Q_{4} e_{5}-e_{6}{ }^{\mathrm{T}} Q_{5} e_{6}-e_{7}{ }^{\mathrm{T}}\left(\varepsilon_{1} I\right) e_{7}-e_{8}{ }^{\mathrm{T}}\left(\varepsilon_{2} I\right) e_{8} \\
& -\frac{1}{d_{12}-d_{11}}\left[\begin{array}{c}
e_{2}-e_{3} \\
e_{5}-e_{6}
\end{array}\right]^{\mathrm{T}}\left[\begin{array}{cc}
R_{2} & M_{1} \\
M_{1}^{\mathrm{T}} & R_{2}
\end{array}\right]\left[\begin{array}{l}
e_{2}-e_{3} \\
e_{5}-e_{6}
\end{array}\right] \\
& -\frac{1}{d_{22}-d_{21}}\left[\begin{array}{l}
e_{3}-e_{4} \\
e_{4}-e_{5}
\end{array}\right]^{\mathrm{T}}\left[\begin{array}{cc}
R_{2} & M_{2} \\
M_{2}{ }^{\mathrm{T}} & R_{2}
\end{array}\right]\left[\begin{array}{l}
e_{3}-e_{4} \\
e_{4}-e_{5}
\end{array}\right]
\end{aligned}
$$

with

$$
\begin{gathered}
\tilde{R}=\left(d_{11}+d_{21}\right)^{2} R_{1}+\left[\left(d_{12}-d_{11}\right)+\left(d_{22}-d_{21}\right)\right] R_{2}, \\
e_{i}=\left[\begin{array}{ccc}
0_{n \times(i-1) n} & I_{n} & 0_{n \times(8-i) n}
\end{array}\right](i=1,2, \cdots, 8), \\
e_{0}=A e_{1}+B e_{4}+e_{7}+e_{8} .
\end{gathered}
$$

Proof Let us consider the following Lyapunov functional as

$$
V(t)=V_{1}(t)+V_{2}(t)+V_{3}(t)
$$

where

$$
\begin{gathered}
V_{1}(t)=x^{\mathrm{T}}(t) P x(t), \\
V_{2}(t)=\int_{t-d_{11}-d_{21}}^{t} x^{\mathrm{T}}(s) Q_{1} x(s) \mathrm{d} s+\int_{t-d_{1}(t)-d_{21}}^{t} x^{\mathrm{T}}(s) Q_{2} x(s) \mathrm{d} s+\int_{t-d_{1}(t)-d_{2}(t)}^{t} x^{\mathrm{T}}(s) Q_{3} x(s) \mathrm{d} s \\
+\int_{t-d_{1}(t)-d_{22}}^{t} x^{\mathrm{T}}(s) Q_{4} x(s) \mathrm{d} s+\int_{t-d_{12}-d_{22}}^{t} x^{\mathrm{T}}(s) Q_{5} x(s) \mathrm{d} s, \\
V_{3}(t)=\left(d_{11}+d_{21}\right) \int_{-d_{11}-d_{21}}^{0} \int_{t+\theta}^{t} \dot{x}^{\mathrm{T}}(s) R_{1} \dot{x}(s) \mathrm{d} s \mathrm{~d} \theta+\int_{-d_{12}-d_{22}}^{-d_{11}-d_{21}} \int_{t+\theta}^{t} \dot{x}^{\mathrm{T}}(s) R_{2} \dot{x}(s) \mathrm{d} s \mathrm{~d} \theta .
\end{gathered}
$$

Along the solution of the system (1), the time derivatives of $V_{1}(t), V_{2}(t)$ and $V_{3}(t)$ can be calculated as

$$
\begin{gathered}
\dot{V}_{1}(t)=2 x^{\mathrm{T}}(t) P\left[A x(t)+B x\left(t-d_{1}(t)-d_{2}(t)\right)+f+g\right] \\
=\xi^{\mathrm{T}}(t)\left(e_{1}^{\mathrm{T}} P e_{0}+e_{0}{ }^{\mathrm{T}} P e_{1}\right) \xi(t) . \\
\dot{V}_{2}(t)=x^{\mathrm{T}}(t)\left(Q_{1}+Q_{2}+Q_{3}+Q_{4}+Q_{5}\right) x(t)-x^{\mathrm{T}}\left(t-d_{11}-d_{21}\right) Q_{1} x\left(t-d_{11}-d_{21}\right) \\
-\left(1-\dot{d}_{1}(t)\right) x^{\mathrm{T}}\left(t-d_{1}(t)-d_{21}\right) Q_{2} x\left(t-d_{1}(t)-d_{21}\right) \\
-\left(1-\dot{d}_{1}(t)-\dot{d}_{2}(t)\right) x^{\mathrm{T}}\left(t-d_{1}(t)-d_{2}(t)\right) Q_{3} x\left(t-d_{1}(t)-d_{2}(t)\right) \\
-\left(1-\dot{d}_{1}(t)\right) x^{\mathrm{T}}\left(t-d_{1}(t)-d_{22}\right) Q_{4} x\left(t-d_{1}(t)-d_{22}\right) \\
-x^{\mathrm{T}}\left(t-d_{12}-d_{22}\right) Q_{5} x\left(t-d_{12}-d_{22}\right) \\
\leq x^{\mathrm{T}}(t)\left(Q_{1}+Q_{2}+Q_{3}+Q_{4}+Q_{5}\right) x(t)-x^{\mathrm{T}}\left(t-d_{11}-d_{21}\right) Q_{1} x\left(t-d_{11}-d_{21}\right) \\
-\left(1-\mu_{1}\right) x^{\mathrm{T}}\left(t-d_{1}(t)-d_{21}\right) Q_{2} x\left(t-d_{1}(t)-d_{21}\right) \\
-\left(1-\mu_{1}-\mu_{2}\right) x^{\mathrm{T}}\left(t-d_{1}(t)-d_{2}(t)\right) Q_{3} x\left(t-d_{1}(t)-d_{2}(t)\right) \\
-\left(1-\mu_{1}\right) x^{\mathrm{T}}\left(t-d_{1}(t)-d_{22}\right) Q_{4} x\left(t-d_{1}(t)-d_{22}\right) \\
-x^{\mathrm{T}}\left(t-d_{12}-d_{22}\right) Q_{5} x\left(t-d_{12}-d_{22}\right) \\
=\xi^{\mathrm{T}}(t)\left[\left(e_{1}^{\mathrm{T}}\left(Q_{1}+Q_{2}+Q_{3}+Q_{4}+Q_{5}\right) e_{1}-e_{2}^{\mathrm{T}} Q_{1} e_{2}-\left(1-\mu_{1}\right) e_{3}^{\mathrm{T}} Q_{2} e_{3}\right.\right. \\
\left.-\left(1-\mu_{1}-\mu_{2}\right) e_{4}^{\mathrm{T}} Q_{3} e_{4}-\left(1-\mu_{1}\right) e_{5}^{\mathrm{T}} Q_{4} e_{5}-e_{6}^{\mathrm{T}} Q_{5} e_{6}\right] \xi(t) .
\end{gathered}
$$




$$
\begin{gathered}
\dot{V}_{3}(t)=\left(d_{11}+d_{21}\right)^{2} \dot{x}^{\mathrm{T}}(t) R_{1} \dot{x}(t)-\left(d_{11}+d_{21}\right) \int_{t-d_{11}-d_{21}}^{t} \dot{x}^{\mathrm{T}}(s) R_{1} \dot{x}(s) \mathrm{d} s \\
+\left[\left(d_{12}-d_{11}\right)+\left(d_{22}-d_{21}\right)\right] \dot{x}^{\mathrm{T}}(t) R_{2} \dot{x}(t)-\int_{t-d_{12}-d_{22}}^{t-d_{11}-d_{21}} \dot{x}^{\mathrm{T}}(s) R_{2} \dot{x}(s) \mathrm{d} s \\
=\dot{x}^{\mathrm{T}}(t) \tilde{R} \dot{x}(t)-\left(d_{11}+d_{21}\right) \int_{t-d_{11}-d_{21}}^{t} \dot{x}^{\mathrm{T}}(s) R_{1} \dot{x}(s) \mathrm{d} s-\int_{t-d_{1}(t)-d_{21}}^{t-d_{11}-d_{21}} \dot{x}^{\mathrm{T}}(s) R_{2} \dot{x}(s) \mathrm{d} s \\
-\int_{t-d_{12}-d_{22}}^{t-d_{1}(t)-d_{22}} \dot{x}^{\mathrm{T}}(s) R_{2} \dot{x}(s) \mathrm{d} s-\int_{t-d_{1}(t)-d_{2}(t)}^{t-d_{1}(t)-d_{21}} \dot{x}^{\mathrm{T}}(s) R_{2} \dot{x}(s) \mathrm{d} s \\
-\int_{t-d_{1}(t)-d_{22}}^{t-d_{1}(t)-d_{2}(t)} \dot{x}^{\mathrm{T}}(s) R_{2} \dot{x}(s) \mathrm{d} s .
\end{gathered}
$$

By the use of Lemma 1, $\dot{V}_{3}(t)$ is bounded as

$$
\begin{gathered}
\dot{V}_{3}(t) \leq \dot{x}^{\mathrm{T}}(t) \tilde{R} \dot{x}(t)-\left[x(t)-x\left(t-d_{11}-d_{21}\right)\right]^{\mathrm{T}} R_{1}\left[x(t)-x\left(t-d_{11}-d_{21}\right)\right] \\
-\frac{1}{d_{1}(t)-d_{11}}\left[x\left(t-d_{11}-d_{21}\right)-x\left(t-d_{1}(t)-d_{21}\right)\right]^{\mathrm{T}} R_{2} \\
\times\left[x\left(t-d_{11}-d_{21}\right)-x\left(t-d_{1}(t)-d_{21}\right)\right] \\
-\frac{1}{d_{12}-d_{1}(t)}\left[x\left(t-d_{1}(t)-d_{22}\right)-x\left(t-d_{12}-d_{22}\right)\right]^{\mathrm{T}} R_{2} \\
\times\left[x\left(t-d_{1}(t)-d_{22}\right)-x\left(t-d_{12}-d_{22}\right)\right] \\
-\frac{1}{d_{2}(t)-d_{21}}\left[x\left(t-d_{1}(t)-d_{21}\right)-x\left(t-d_{1}(t)-d_{2}(t)\right)\right]^{\mathrm{T}} R_{2} \\
\quad \times\left[x\left(t-d_{1}(t)-d_{21}\right)-x\left(t-d_{1}(t)-d_{2}(t)\right)\right] \\
-\frac{1}{d_{22}-d_{2}(t)}\left[x\left(t-d_{1}(t)-d_{2}(t)\right)-x\left(t-d_{1}(t)-d_{22}\right)\right]^{\mathrm{T}} R_{2} \\
\quad \times\left[x\left(t-d_{1}(t)-d_{2}(t)\right)-x\left(t-d_{1}(t)-d_{22}\right)\right] \\
=\xi^{\mathrm{T}}(t) e_{0}^{\mathrm{T}} \tilde{R} e_{0} \xi(t)-\xi^{\mathrm{T}}(t)\left(e_{1}-e_{2}\right)^{\mathrm{T}} R_{1}\left(e_{1}-e_{2}\right) \xi(t) \\
-\frac{1}{d_{12}-d_{11}} \frac{1}{\gamma_{11}} \xi^{\mathrm{T}}(t)\left(e_{2}-e_{3}\right)^{\mathrm{T}} R_{2}\left(e_{2}-e_{3}\right) \xi(t) \\
-\frac{1}{d_{12}-d_{11}} \frac{1}{\gamma_{12}} \xi^{\mathrm{T}}(t)\left(e_{5}-e_{6}\right)^{\mathrm{T}} R_{2}\left(e_{5}-e_{6}\right) \xi(t) \\
-\frac{1}{d_{22}-d_{21}} \frac{1}{\gamma_{21}} \xi^{\mathrm{T}}(t)\left(e_{3}-e_{4}\right)^{\mathrm{T}} R_{2}\left(e_{3}-e_{4}\right) \xi(t) \\
-\frac{1}{d_{22}-d_{21}} \frac{1}{\gamma_{22}} \xi^{\mathrm{T}}(t)\left(e_{4}-e_{5}\right)^{\mathrm{T}} R_{2}\left(e_{4}-e_{5}\right) \xi(t),
\end{gathered}
$$

where

$$
\gamma_{11}=\frac{d_{1}(t)-d_{11}}{d_{12}-d_{11}}, \gamma_{12}=\frac{d_{12}-d_{1}(t)}{d_{12}-d_{11}}, \gamma_{21}=\frac{d_{2}(t)-d_{21}}{d_{22}-d_{21}}, \gamma_{22}=\frac{d_{22}-d_{2}(t)}{d_{22}-d_{21}} .
$$

Furthermore, from LMI (6) and applying Lemma 2 leads to 


$$
\begin{aligned}
\dot{V}_{3}(t) & \leq \xi^{\mathrm{T}}(t) e_{0}^{\mathrm{T}} \tilde{R} e_{0} \xi(t)-\xi^{\mathrm{T}}(t)\left(e_{1}-e_{2}\right)^{\mathrm{T}} R_{1}\left(e_{1}-e_{2}\right) \xi(t) \\
& -\frac{1}{d_{12}-d_{11}} \xi^{\mathrm{T}}(t)\left[\begin{array}{c}
e_{2}-e_{3} \\
e_{5}-e_{6}
\end{array}\right]^{\mathrm{T}}\left[\begin{array}{cc}
R_{2} & M_{1} \\
M_{1}^{\mathrm{T}} & R_{2}
\end{array}\right]\left[\begin{array}{l}
e_{2}-e_{3} \\
e_{5}-e_{6}
\end{array}\right] \xi(t) \\
& -\frac{1}{d_{22}-d_{21}} \xi^{\mathrm{T}}(t)\left[\begin{array}{l}
e_{3}-e_{4} \\
e_{4}-e_{5}
\end{array}\right]^{\mathrm{T}}\left[\begin{array}{cc}
R_{2} & M_{2} \\
M_{2}^{\mathrm{T}} & R_{2}
\end{array}\right]\left[\begin{array}{l}
e_{3}-e_{4} \\
e_{4}-e_{5}
\end{array}\right] \xi(t) .
\end{aligned}
$$

On the other hand, from (3) and (4), it yields that

$$
\begin{aligned}
0 & \leq \varepsilon_{1}\left[\alpha^{2} x^{\mathrm{T}}(t) F^{\mathrm{T}} F x(t)-f^{\mathrm{T}} f\right] \\
& =\xi^{\mathrm{T}}(t)\left[e_{1}^{\mathrm{T}}\left(\varepsilon_{1} \alpha^{2} F^{\mathrm{T}} F\right) e_{1}-e_{7}^{\mathrm{T}}\left(\varepsilon_{1} I\right) e_{7}\right] \xi(t)
\end{aligned}
$$

and

$$
\begin{aligned}
0 & \leq \varepsilon_{2}\left[\beta^{2} x^{\mathrm{T}}\left(t-d_{1}(t)-d_{2}(t)\right) G^{\mathrm{T}} G x\left(t-d_{1}(t)-d_{2}(t)\right)-g^{\mathrm{T}} g\right] \\
& =\xi^{\mathrm{T}}(t)\left[e_{4}^{\mathrm{T}}\left(\varepsilon_{2} \beta^{2} G^{\mathrm{T}} G\right) e_{4}-e_{8}^{\mathrm{T}}\left(\varepsilon_{2} I\right) e_{8}\right] \xi(t) .
\end{aligned}
$$

Taking account of (8)-(12), we get

$$
\dot{V}(t) \leq \xi^{\mathrm{T}}(t) \Xi \xi(t) .
$$

It's clear that if (5) and (13) hold, then $\dot{V}(t)<0$ for any $\xi(t) \neq 0$. This implies $\dot{V}(t) \leq-\lambda\|x(t)\|^{2}$ for sufficiently small $\lambda>0$, and ensures that the system (1) is stable. This completes the proof.

Remark 1 In many circumstance, the information on the delay derivative may not be available, for this case, the corresponding result can be obtained from Theorem 1 by setting $Q_{2}=Q_{3}=Q_{4}=0$. Here, it is omitted for brevity.

Remark 2 The norm-bounded uncertainties can be treated as a special case of nonlinear perturbations $[8,14]$. Therefore, the criterion obtained in Theorem 1 can be applicable to the system with two additive interval time-varying delays and normbounded uncertainties.

If $f=0$ and $g=0$, that is, there is no perturbation, the stability criterion can be readily deduced from Theorem 1 . It is presented in the following corollary.

Corollary 1 For given scalars $0 \leq d_{i 1}<d_{i 2}, 0 \leq \mu_{i}<1(i=1,2)$, the system (1) with two additive interval time-varying delays $d_{1}(t)$ and $d_{2}(t)$ satisfying (2) and nonlinear perturbations $f=g=0$ is stable, if there exist matrices $P>0, Q_{i}>0(i=1,2, \cdots, 5)$, $R_{j}>0$ and $M_{j}(i=1,2)$ such that the LMI (6) and following LMI hold

where

$$
\tilde{\Xi}<0 \text {, }
$$

$$
\begin{aligned}
& \tilde{\Xi}=\tilde{e}_{0}^{\mathrm{T}} \tilde{R} \tilde{e}_{0}+\tilde{e}_{0}^{\mathrm{T}} P \tilde{e}_{1}+\tilde{e}_{1}^{\mathrm{T}} P \tilde{e}_{0}+\tilde{e}_{1}^{\mathrm{T}}\left(Q_{1}+Q_{2}+Q_{3}+Q_{4}+Q_{5}\right) \tilde{e}_{1}-\left(\tilde{e}_{1}-\tilde{e}_{2}\right)^{\mathrm{T}} R_{1}\left(\tilde{e}_{1}-\tilde{e}_{2}\right) \\
& -\tilde{e}_{2}{ }^{\mathrm{T}} Q_{1} \tilde{e}_{2}-\left(1-\mu_{1}\right) \tilde{e}_{3}^{\mathrm{T}} Q_{2} \tilde{e}_{3}-\left(1-\mu_{1}-\mu_{2}\right) \tilde{e}_{4}^{\mathrm{T}} Q_{3} \tilde{e}_{4}-\left(1-\mu_{1}\right) \tilde{e}_{5}^{\mathrm{T}} Q_{4} \tilde{e}_{5}-\tilde{e}_{6}{ }^{\mathrm{T}} Q_{5} \tilde{e}_{6} \\
& -\frac{1}{d_{12}-d_{11}}\left[\begin{array}{c}
\tilde{e}_{2}-\tilde{e}_{3} \\
\tilde{e}_{5}-\tilde{e}_{6}
\end{array}\right]^{\mathrm{T}}\left[\begin{array}{cc}
R_{2} & M_{1} \\
M_{1}^{\mathrm{T}} & R_{2}
\end{array}\right]\left[\begin{array}{c}
\tilde{e}_{2}-\tilde{e}_{3} \\
\tilde{e}_{5}-\tilde{e}_{6}
\end{array}\right] \\
& -\frac{1}{d_{22}-d_{21}}\left[\begin{array}{c}
\tilde{e}_{3}-\tilde{e}_{4} \\
\tilde{e}_{4}-\tilde{e}_{5}
\end{array}\right]^{\mathrm{T}}\left[\begin{array}{cc}
R_{2} & M_{2} \\
M_{2}{ }^{\mathrm{T}} & R_{2}
\end{array}\right]\left[\begin{array}{l}
\tilde{e}_{3}-\tilde{e}_{4} \\
\tilde{e}_{4}-\tilde{e}_{5}
\end{array}\right],
\end{aligned}
$$


with

$$
\begin{gathered}
\tilde{R}=\left(d_{11}+d_{21}\right)^{2} R_{1}+\left[\left(d_{12}-d_{11}\right)+\left(d_{22}-d_{21}\right)\right] R_{2}, \\
\tilde{e}_{i}=\left[\begin{array}{ccl}
0_{n \times(i-1) n} & I_{n} & 0_{n \times(6-i) n}
\end{array}\right](i=1,2, \cdots, 6), \\
\tilde{e}_{0}=A \tilde{e}_{1}+B \tilde{e}_{4} .
\end{gathered}
$$

If $f=g=0$ and $d_{11}=d_{21}=0$, then the system (1) reduces to the following system

$$
\left\{\begin{array}{l}
\dot{x}=A x(t)+B x\left(t-d_{1}(t)-d_{2}(t)\right), \\
x(t)=\varphi(t), t \in\left[-h_{1}-h_{2}, 0\right] .
\end{array}\right.
$$

Assuming time-varying delays $d_{1}(t)$ and $d_{2}(t)$ satisfy following conditions

$$
0 \leq d_{i}(t) \leq h_{i}, \dot{d}_{i}(t) \leq \mu_{i},(i=1,2)
$$

where $h_{i}>0,0 \leq \mu_{i}<1(i=1,2)$ are known constants. The stability problem of system (15) has been widely studied in the recent literature (see, e.g.[11-19]). It is worth pointing out that there has a technical problem in the Theorem 3.1 and proof in [17] (see [20]). In order to estimate the upper bound of the derivative of Lyapunov functional, [17] apply the following bounding inequalities

$$
\begin{aligned}
& -\left(1-\dot{d}_{1}(t)\right) x^{\mathrm{T}}\left(t-d_{1}(t)\right)\left(Q_{2}-Q_{7}\right) x\left(t-d_{1}(t)\right) \\
& \leq-\left(1-\mu_{1}\right) x^{\mathrm{T}}\left(t-d_{1}(t)\right)\left(Q_{2}-Q_{7}\right) x\left(t-d_{1}(t)\right), \\
& -\left(1-\dot{d}_{2}(t)\right) x^{\mathrm{T}}\left(t-d_{2}(t)\right)\left(Q_{5}-Q_{8}\right) x\left(t-d_{2}(t)\right) \\
& \leq-\left(1-\mu_{2}\right) x^{\mathrm{T}}\left(t-d_{2}(t)\right)\left(Q_{5}-Q_{8}\right) x\left(t-d_{2}(t)\right),
\end{aligned}
$$

where $Q_{2}>0, Q_{5}>0, Q_{7}>0$ and $Q_{8}>0$. Obviously, inequalities (17) and (18) hold only on conditions that $Q_{2} \geq Q_{7}$ and $Q_{5} \geq Q_{8}$, respectively. However, the matrix constraints $Q_{2} \geq Q_{7}$ and $Q_{5} \geq Q_{8}$ were neglected in Theorem 3.1 in [17]. The corrected result stated in the following proposition.

Proposition 1 For given scalars $h_{i}>0,0 \leq \mu_{i}<1(i=1,2)$, the system (15) with two additive time-varying delays $d_{1}(t)$ and $d_{2}(t)$ satisfying (16) is stable, if there exist matrices $P>0, Q_{i}>0(i=1,2, \cdots, 8), R_{j}>0(j=1,2, \cdots, 5)$, and $X_{k}(k=1,2,3)$, such that the following LMIs hold

$$
\begin{gathered}
\Theta_{k}=\left[\begin{array}{cc}
R_{k} & X_{k}^{\mathrm{T}} \\
X_{k} & R_{k}
\end{array}\right] \geq 0(k=1,2,3) \\
Q_{2}-Q_{7} \geq 0, \\
Q_{5}-Q_{8} \geq 0,
\end{gathered}
$$

where 


$$
\begin{aligned}
& \Gamma=\kappa_{1}\left(P A+A^{\mathrm{T}} P+\sum_{i=1}^{6} Q_{i}\right) \kappa_{1}^{\mathrm{T}}+\kappa_{1}\left(P B+B^{\mathrm{T}} P\right) \kappa_{3}^{\mathrm{T}}-\left(1-\mu_{1}\right) \kappa_{2}\left(Q_{2}-Q_{7}\right) \kappa_{2}^{\mathrm{T}} \\
& -\left(1-\mu_{1}-\mu_{2}\right) \kappa_{3}\left(Q_{1}+Q_{7}+Q_{8}\right) \kappa_{3}{ }^{\mathrm{T}}-\kappa_{5} Q_{3} \kappa_{5}{ }^{\mathrm{T}}-\kappa_{4} Q_{4} \kappa_{4}{ }^{\mathrm{T}} \\
& -\left(1-\mu_{2}\right) \kappa_{6}\left(Q_{5}-Q_{8}\right) \kappa_{6}^{\mathrm{T}}-\kappa_{7} Q_{6} \kappa_{7}^{\mathrm{T}} \\
& +\left(A \kappa_{1}^{\mathrm{T}}+B \kappa_{3}^{\mathrm{T}}\right)^{\mathrm{T}}\left[\left(h_{1}+h_{2}\right)^{2} R_{1}+h_{1}{ }^{2}\left(R_{2}+R_{5}\right)+h_{2}{ }^{2}\left(R_{3}+R_{4}\right)\right]\left(A \kappa_{1}^{\mathrm{T}}+B \kappa_{3}^{\mathrm{T}}\right) \\
& -\left[\begin{array}{c}
\left(\kappa_{1}-\kappa_{3}\right)^{\mathrm{T}} \\
\left(\kappa_{3}-\kappa_{5}\right)^{\mathrm{T}}
\end{array}\right]^{\mathrm{T}} \Theta_{1}\left[\begin{array}{c}
\left(\kappa_{1}-\kappa_{3}\right)^{\mathrm{T}} \\
\left(\kappa_{3}-\kappa_{5}\right)^{\mathrm{T}}
\end{array}\right]-\left[\begin{array}{c}
\left(\kappa_{1}-\kappa_{2}\right)^{\mathrm{T}} \\
\left(\kappa_{2}-\kappa_{4}\right)^{\mathrm{T}}
\end{array}\right]^{\mathrm{T}} \Theta_{2}\left[\begin{array}{l}
\left(\kappa_{1}-\kappa_{2}\right)^{\mathrm{T}} \\
\left(\kappa_{2}-\kappa_{4}\right)^{\mathrm{T}}
\end{array}\right] \\
& -\left[\begin{array}{c}
\left(\kappa_{1}-\kappa_{6}\right)^{\mathrm{T}} \\
\left(\kappa_{6}-\kappa_{7}\right)^{\mathrm{T}}
\end{array}\right]^{\mathrm{T}} \Theta_{3}\left[\begin{array}{c}
\left(\kappa_{1}-\kappa_{6}\right)^{\mathrm{T}} \\
\left(\kappa_{6}-\kappa_{7}\right)^{\mathrm{T}}
\end{array}\right]-\left(\kappa_{4}-\kappa_{5}\right) R_{4}\left(\kappa_{4}-\kappa_{5}\right)^{\mathrm{T}} \\
& -\left(\kappa_{7}-\kappa_{5}\right) R_{5}\left(\kappa_{7}-\kappa_{5}\right)^{\mathrm{T}} \text {, }
\end{aligned}
$$

with

$$
\kappa_{i}=\left[\begin{array}{lll}
0_{n \times(i-1) n} & I_{n} & 0_{n \times(7-i) n}
\end{array}\right](i=1,2, \cdots, 7) .
$$

In the following, we present a new stability criterion for the system (15). By simplifying the Lyapunov functional (7) used in Theorem 1 to

$$
\begin{aligned}
V(t) & =x^{\mathrm{T}}(t) P x(t)+\int_{t-d_{1}(t)}^{t} x^{\mathrm{T}}(s) Q_{1} x(s) \mathrm{d} s+\int_{t-d_{1}(t)-d_{2}(t)}^{t} x^{\mathrm{T}}(s) Q_{2} x(s) \mathrm{d} s \\
& +\int_{t-d_{1}(t)-h_{2}}^{t} x^{\mathrm{T}}(s) Q_{3} x(s) \mathrm{d} s+\int_{t-h_{1}-h_{2}}^{t} x^{\mathrm{T}}(s) Q_{4} x(s) \mathrm{d} s \\
& +\int_{-h_{1}-h_{2}}^{0} \int_{t+\theta}^{t} \dot{x}^{\mathrm{T}}(s) R \dot{x}(s) \mathrm{d} s \mathrm{~d} \theta,
\end{aligned}
$$

where $P>0, Q_{i}>0(i=1,2,3,4)$ and $R>0$, following the same lines as in the proof of Theorem 1, we can obtain the following delay-dependent stability criterion for systems (15).

Corollary 2 For given scalars $h_{i}>0,0 \leq \mu_{i}<1(i=1,2)$, the system (15) with two additive interval time-varying delays $d_{1}(t)$ and $d_{2}(t)$ satisfying (16) is stable, if there exist matrices $P>0, Q_{i}>0(i=1,2,3,4), R>0$ and $M_{j}(i=1,2)$, such that the LMI (6) and the following LMI hold

$$
\Gamma<0
$$

where

$$
\begin{aligned}
& \Gamma=\left(h_{1}+h_{2}\right) \hat{e}_{0}^{\mathrm{T}} R \hat{e}_{0}+\hat{e}_{0}^{\mathrm{T}} P \hat{e}_{1}+\hat{e}_{1}^{\mathrm{T}} P \hat{e}_{0}+\hat{e}_{1}^{\mathrm{T}}\left(Q_{1}+Q_{2}+Q_{3}+Q_{4}\right) \hat{e}_{1} \\
& -\left(1-\mu_{1}\right) \hat{e}_{2}^{\mathrm{T}} Q_{1} \hat{e}_{2}-\left(1-\mu_{1}-\mu_{2}\right) \hat{e}_{3}^{\mathrm{T}} Q_{2} \hat{e}_{3}-\left(1-\mu_{1}\right) \hat{e}_{4}^{\mathrm{T}} Q_{3} \hat{e}_{4}-\hat{e}_{5}^{\mathrm{T}} Q_{4} \hat{e}_{5} \\
& -\frac{1}{h_{1}}\left[\begin{array}{c}
\hat{e}_{1}-\hat{e}_{2} \\
\hat{e}_{4}-\hat{e}_{5}
\end{array}\right]^{\mathrm{T}}\left[\begin{array}{cc}
R & M_{1} \\
M_{1}^{\mathrm{T}} & R
\end{array}\right]\left[\begin{array}{c}
\hat{e}_{1}-\hat{e}_{2} \\
\hat{e}_{4}-\hat{e}_{5}
\end{array}\right]-\frac{1}{h_{2}}\left[\begin{array}{c}
\hat{e}_{2}-\hat{e}_{3} \\
\hat{e}_{3}-\hat{e}_{4}
\end{array}\right]^{\mathrm{T}}\left[\begin{array}{cc}
R & M_{2} \\
M_{2}^{\mathrm{T}} & R
\end{array}\right]\left[\begin{array}{l}
\hat{e}_{2}-\hat{e}_{3} \\
\hat{e}_{3}-\hat{e}_{4}
\end{array}\right],
\end{aligned}
$$

with

$$
\begin{gathered}
\hat{e}_{i}=\left[\begin{array}{ccc}
0_{n \times(i-1) n} & I_{n} & 0_{n \times(5-i) n}
\end{array}\right](i=1,2, \cdots, 5), \\
\hat{e}_{0}=A \hat{e}_{1}+B \hat{e}_{3} .
\end{gathered}
$$

Remark 3 Based on the Lyapunov functional (23), Corollary 2 proposed a new delaydependent stability criterion for system (15). It is noted that unlike the Lyapunov functionals chosen in [11-19], the constructed Lyapunov functional (23) takes the 
information of delay state $x\left(t-d_{1}(t)-h_{2}\right)$ into account. Therefore, Corollary 2 is expected to be less conservative than the ones in [11-19], which will be demonstrated in the sequel.

Remark 4 It is worth mentioning that in $[11-16,18]$, too many free-weighting matrices were introduced to discuss the stability problem for system (15). However, some matrices do not reduce the conservatism of the resulting criterion, on the contrary, they make the analysis more complicated. While in our discussion, we enlarge the cross terms appeared in the derivative of Lyapunov functional by introducing two free-weighting matrices, i.e. $M_{1}$ and $M_{2}$. Thus, the criterion in Corollary 2 has less decision variables than the ones in $[11-16,18]$.

\section{Numerical Examples}

In the section, numerical examples are given to illustrate the effectiveness of obtained results in this paper.

Example 1 Consider the system (1) with following parameters

$$
A=\left[\begin{array}{cc}
-2.0 & 0 \\
0 & -0.9
\end{array}\right], B=\left[\begin{array}{cc}
-1.0 & 0 \\
-1.0 & -1.0
\end{array}\right], F=G=\left[\begin{array}{cc}
1.0 & 0 \\
0 & 1.0
\end{array}\right] \text {. }
$$

Let $d_{11}=d_{21}=0.1, \mu_{1}=0.1, \mu_{2}=0.5$. Our purpose is to calculate the maximum allowable upper delay bound $d_{22}$ of delay $d_{2}(t)$, or $d_{12}$ of delay $d_{1}(t)$, when the other is known, below which the system (1) is stable. The maximum allowable upper delay bound $d_{22}$ obtained by Theorem 1 or Corollary 1 in this paper for different $d_{12}, \alpha$ and $\beta$ are listed in Table 1. The maximum allowable upper delay bound $d_{12}$ obtained by Theorem 1 or Corollary 1 in this paper for different $d_{22}, \alpha$ and $\beta$ are listed in Table 2. We can see the effectiveness of the method proposed in this paper. 
Table 1. Allowable Upper Bound of $d_{22}$ for different $d_{12}, \alpha$ and $\beta$

\begin{tabular}{|c|c|c|c|c|c|}
\hline Methods & $\alpha$ & $\beta$ & $d_{12}=0.9$ & $d_{12}=1.2$ & $d_{12}=1.5$ \\
\hline Theorem 1 & 0.2 & 0.1 & 0.5519 & 0.3235 & 0.1437 \\
\hline Theorem 1 & 0.1 & 0.1 & 0.6725 & 0.4519 & 0.2721 \\
\hline Corollary 1 & 0 & 0 & 1.2699 & 0.9699 & 0.7296 \\
\hline
\end{tabular}

Table 2.Allowable Upper Bound of $d_{12}$ for different $d_{22}, \alpha$ and $\beta$

\begin{tabular}{|c|c|c|c|c|c|}
\hline Methods & $\alpha$ & $\beta$ & $d_{22}=0.2$ & $d_{22}=0.5$ & $d_{22}=0.8$ \\
\hline Theorem 1 & 0.2 & 0.1 & 1.3958 & 0.9628 & 0.6308 \\
\hline Theorem 1 & 0.1 & 0.1 & 1.6442 & 1.1294 & 0.7572 \\
\hline Corollary 1 & 0 & 0 & 2.9594 & 1.9763 & 1.3952 \\
\hline
\end{tabular}

Example 2 Consider the system (15) with following parameters

$$
A=\left[\begin{array}{cc}
-2.0 & 0 \\
0 & -0.9
\end{array}\right], B=\left[\begin{array}{cc}
-1.0 & 0 \\
-1.0 & -1.0
\end{array}\right] \text {. }
$$

Let $\mu_{1}=0.1, \mu_{2}=0.8$. Our purpose is to calculate the maximum allowable upper delay bound $h_{2}$ of delay $d_{2}(t)$, or $h_{1}$ of delay $d_{1}(t)$, when the other is known, below which the system (15) is stable. The calculation results obtained by [11, 12, 14-16, 18, 19 ] and Proposition 1, Corollary 2 in this paper are listed in Table 3 and Table 4. Meanwhile, Table 5 provides a comparison of the number of the decision variables involved in Corollary 2 and [11-19].

Table 3. Allowable Upper Bound of ${ }^{h_{2}}$ for different $h_{1}$

\begin{tabular}{|c|c|c|c|}
\hline Methods & $h_{1}=1.0$ & $h_{1}=1.2$ & $h_{1}=1.5$ \\
\hline$[11]$ & 0.415 & 0.340 & 0.248 \\
\hline$[12]$ & 0.512 & 0.406 & 0.283 \\
\hline$[14]$ & 0.872 & 0.672 & 0.371 \\
\hline Proposition 1, [19] & 0.873 & 0.673 & 0.373 \\
\hline$[15,16]$ & 0.873 & 0.673 & 0.452 \\
\hline$[18]$ & 0.583 & 0.519 & 0.421 \\
\hline Corollary 2 & 0.9883 & 0.8546 & 0.6753 \\
\hline
\end{tabular}


Table 4. Allowable Upper Bound of ${ }^{h_{1}}$ for different $h_{2}$

\begin{tabular}{|c|c|c|c|}
\hline Methods & $h_{2}=0.1$ & $h_{2}=0.2$ & $h_{2}=0.3$ \\
\hline$[11]$ & 2.263 & 1.696 & 1.324 \\
\hline$[12]$ & 2.300 & 1.779 & 1.453 \\
\hline$[14]$ & 1.772 & 1.672 & 1.572 \\
\hline Proposition 1, [19] & 1.773 & 1.673 & 1.573 \\
\hline$[15,16]$ & 2.558 & 2.100 & 1.808 \\
\hline$[18]$ & 2.997 & 2.332 & 1.899 \\
\hline Corollary 2 & 3.0579 & 2.6488 & 2.3324 \\
\hline
\end{tabular}

Table 5. Number of Decision Variables

\begin{tabular}{|c|c|}
\hline Methods & Number of decision variables \\
\hline$[11]$ & $12.5 n^{2}+4.5 n$ \\
\hline$[12]$ & $19.5 n^{2}+3.5 n$ \\
\hline$[13,15]$ & $15.5 n^{2}+3.5 n$ \\
\hline$[14]$ & $32.5 n^{2}+8.5 n$ \\
\hline$[16]$ & $8.5 n^{2}+2.5 n$ \\
\hline$[17]$ & $10 n^{2}+7 n$ \\
\hline$[18]$ & $22.5 n^{2}+10.5 n$ \\
\hline$[19]$ & $7 n^{2}+5 n$ \\
\hline Corollary 2 & $5 n^{2}+3 n$ \\
\hline
\end{tabular}

From Table 3-5, one can see that: (i) The maximum allowable upper delay bound provided by Theorem 3.1 in [17] are incorrect, which are corrected by Proposition 1. (ii) Corollary 2 in this paper give much better results than those obtained by some recently report results [11-12, 14-19]. (iii) Corollary 2 in this paper contain fewer decision variables than those conditions in [11-19], hence, it is mathematically less complex and computationally more efficient.

\section{Conclusion}

This paper deals with the problem of delay-dependent stability for continuous systems with two additive interval time-varying delays and nonlinear perturbations. By constructing a suitable Lyapunov functional, and using reciprocally convex technique, delay-dependent stability criteria are proposed in terms of LMIs for considered systems. Based on the obtained criteria, an improved stability result for continuous systems with two additive time-varying delays is also given. These results have been shown to be less conservative than some recently report results. Moreover, the proposed method decreases the computational complexity comparable to some existing methods. Two numerical examples are given to illustrate the applicability of the results. 


\section{Acknowledgments}

The authors would like to thank all anonymous referees for their helpful suggestions. This research was supported by the Research Foundation of Baoji University of Arts and Sciences (Project No. ZK16010).

\section{References}

[1] G. Keqin, J. Chen, and V L. Kharitonov, "Stability of time-delay systems”, Springer Science \& Business Media, (2003).

[2] E. Fridman and U. Shaked, "Delay-dependent stability and $\mathrm{H}_{\infty}$ control: constant and time-varying delays", International journal of control, vol. 76, no. 1, (2003), pp. 48-60.

[3] Y. He, Q.G. Wang, C. Lin and M. Wu, "Delay-range-dependent stability for systems with time-varying delay", Automatica, vol. 43, no. 2, (2007), pp. 371-376.

[4] H.Y. Shao, "New delay-dependent stability criteria for systems with interval delay", Automatica, vol. 45, no. 3, (2009), pp. 744-749.

[5] P.G. Park, J.W. Ko and C. Jeong, "Reciprocally convex approach to stability of systems with timevarying delays", Automatica, vol. 47, no. 1, (2011), pp. 235-238.

[6] L. Dai, "Singular control system", Springer-Verlag New York, Inc., (1989).

[7] J.M. Jiao, "Improved Stability Criteria for Neural Networks with Additive Time-varying Delay Components", International Journal of Control and Automation, vol. 8, no. 9, (2015), pp. 353-364.

[8] L. Orihuela, P. Millan, C. Vivas and F.R. Rubio, "Robust stability of nonlinear time-delay systems with interval time-varying delay", International Journal of Robust and Nonlinear Control, vol. 21, no. 7, (2011), pp. 709-724.

[9] X.L. Zhu, H. Yang, Y. Wang and Y.L. Wang, "New stability criterion for linear switched systems with time-varying delay", International Journal of Robust and Nonlinear Control, vol. 24, no. 2, (2014), pp. 214-227.

[10] U. Shaked and E. Gershon, "Robust $\mathrm{H}_{\infty}$ control of stochastic linear switched systems with dwell time", International Journal of Robust and Nonlinear Control, vol. 24, no. 11, (2014), pp. 1664-1676.

[11] J. Lam, H.J. Gao and C.H. Wang, "Stability analysis for continuous systems with two additive timevarying delay components", Systems \& Control Letters, vol. 56, no. 1, (2007), pp. 16-24.

[12] H.J. Gao, T.W. Chen and J. Lam, "A new delay system approach to network-based control", Automatica, vol. 44, no. 1, (2008), pp. 39-52.

[13] R. Dey, G. Ray, S. Ghosh and A. Rakshit, "Stability analysis for continuous system with additive timevarying delays: A less conservative result", Applied Mathematics and Computation, vol. 215, no. 10, (2010), pp. 3740-3745.

[14] H.X. Wu, X.F Liao, W. Feng, S.T. Guo and W. Zhang, "Robust stability analysis of uncertain systems with two additive time-varying delay components", Applied Mathematical Modelling, vol. 33, no. 12, (2009), pp. 4345-4353.

[15] H. Y. Shao, X.L. Zhu, Z.Q. Zhang and G.D. Zhong, "Delay-dependent $\mathrm{H}_{\infty}$ control for systems with two additive time-vary delays", Journal of Control Engineering and Technology, vol. 3, no. 2, (2013), pp. 90-97.

[16] J.M. Jiao, "A stability criterion for singular systems with two additive time-varying delay components", International Journal of Automation and Computing, vol. 10, no. 1, (2013), pp. 39-45.

[17] J. Cheng, H. Zhu, S.M. Zhong, Y.P. Zhang and Y. Zeng, "Improved delay-dependent stability criteria for continuous system with two additive time-varying delay components", Communications in Nonlinear Science and Numerical Simulation, vol. 19, no. 1, (2014), pp. 210-215.

[18] P.L. Liu, "Further results on delay-range-dependent stability with additive time-varying delay systems", ISA transactions, vol. 53, no. 2, (2014), pp. 258-266.

[19] X.H. Ge, "Comments and an improved result on 'Stability analysis for continuous system with additive time-varying delays: A less conservative result' ", Applied Mathematics and Computation, vol. 241, (2014), pp. 42-46.

[20] K. Ramakrishnan, V. Venkatachalam and G. Ray, "Comments on 'Improved delay-dependent stability criteria for continuous systems with additive time-varying delays components" ", Communications in Nonlinear Science and Numerical Simulation, vol. 24, no. 1, (2015), pp. 1-3. 


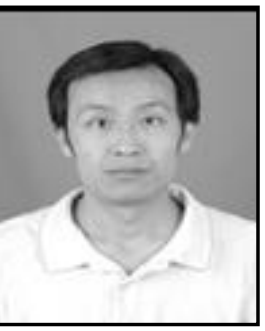

\section{Authors}

Jianmin Jiao, He received the M.S. in College of Mathematics and Information Science from Shaanxi Normal University, Xi'an, China, in 2009. He is currently a associate professor of Institute of Mathematics and Information Science, Baoji University of Arts and Sciences, China. His research interests include time-delay systems, neural networks, singular systems and robust control.

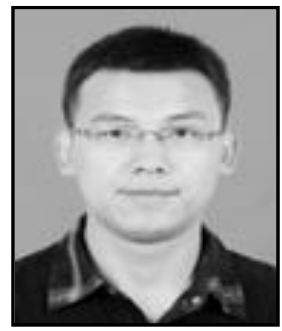

Xiaojun Sun, He received the M.S. in School of Mathematics and Statistics from Xidian University, Xi'an, China, in 2006. He is currently an associate professor of Institute of Mathematics and Information Science, Baoji University of Arts and Sciences, China. His research interests include optimization theory and algorithms, mathematical modeling. 
International Journal of Control and Automation Vol.10, No.4 (2017) 